\title{
DESCONEXÃO NO SISTEMA DE INOVAÇÃO NO SETOR SAÚDE: UMA AVALIAÇÃO PRELIMINAR DO CASO BRASILEIRO A PARTIR DE ESTATÍSTICAS DE PATENTES E ARTIGOS*
}

\author{
Catari Vilela Chaves ${ }^{\S}$ \\ Eduardo da Motta e Albuquerque ${ }^{a}$
}

\begin{abstract}
RESUMO
Este artigo formula uma conjectura sobre o sistema de inovação no setor saúde no Brasil: a existência de uma "desconexão" entre as produções científica e tecnológica. A conjectura é avaliada por meio de estatísticas de patentes e artigos científicos. Essas estatísticas são utilizadas para localizar a posição do Brasil no cenário internacional e para cotejar as especializações de mesorregiões do País em termos de ciência e tecnologia. A desconexão no sistema de inovação do setor saúde investigada no artigo está relacionada à forte presença de patentes da área entre as patentes de não-residentes no Brasil e a problemas na balança comercial do setor.
\end{abstract}

Palavras-chave: economia da tecnologia, sistema de inovação, ciência e tecnologia em saúde.

\begin{abstract}
This paper conjectures about a disconnection between the scientific and the technological dimensions of the Brazilian health innovation system. Statistics of patents and scientific papers are used in the investigation of this conjecture. The international position of Brazil in regard to the health innovation system is identified. The disconnection between the scientific and technological productions in Brazil is investigated at the regional level. Deficits in the sectoral trade balance and the high percentage of health-related non-resident patents seem to be associated with the disconnection conjectured throughout this paper.
\end{abstract}

Key words: economics of technology, innovation system, science and technology in health.

JEL classification: $\mathrm{O} 30, \mathrm{I} 10$.

\footnotetext{
* Agradecimentos para Regina Gusmão (Fapesp) e Sinésio Fernandes (MCT) pela obtenção dos dados de patentes no INPI, para Renato Viotti (MCT) pelos dados do ISI para os anos de 1981, 1991 e 2001 e aos dois pareceristas anônimos da revista Economia Aplicada. Esta pesquisa contou com o apoio da economista Camila Lins e das bolsistas de iniciação científica Cíntia Oliveira, Hérica Righi e Paula Januzzi e se beneficia de resultados de outras pesquisas (anteriores e em curso) que contam com a participação de Leandro Silva e Adriano Baessa. A responsabilidade pelos erros é exclusiva dos autores.

$\S$ Cedeplar-UFMG e Pucminas.E-mail: catari@pucminas.br.

a Cedeplar-UFMG. E-mail: albuquerque@cedeplar.ufmg.br.

Recebido em agosto de 2004. Aprovado em julho de 2006.
} 


\section{INTRODUÇÃo}

Este artigo formula uma conjectura sobre o sistema de inovação no setor saúde no Brasil: a existência de uma "desconexão" entre as produções científica e tecnológica. A conjectura apóia-se em uma avaliação preliminar quantitativa do estágio de construção do sistema de inovação do setor saúde no País. A avaliação utiliza três fontes de dados: artigos científicos indexados pelo Institute for Scientific Information (ISI), patentes registradas no Instituto Nacional de Propriedade Industrial (INPI) e no United States Patent and Trademark Office (USPTO).

A conjectura de existência da "desconexão" é resultado de uma comparação simples entre as estatísticas de artigos científicos com autores brasileiros (ISI) e as de patentes de residentes no Brasil (INPI) entre 1990 e 2001. Por um lado, a participação relativa da área da saúde nesses artigos (ISI) alcança a marca de $46 \%$. Por outro lado, apenas $7 \%$ das patentes de residentes no Brasil (segundo INPI) referem-se a domínios tecnológicos relacionados à saúde. Essa desconexão parece ser uma característica geral de sistemas de inovação imaturos, identificada em outros estudos baseados em estatísticas de artigos e patentes. Em particular, Bernardes et al. (2003) sugerem um papel importante para a existência de "massa crítica" em termos de produção científica para o início de circuitos virtuosos entre ciência e tecnologia, característicos de sistemas de inovação maduros. $\mathrm{O}$ Brasil faz parte de um conjunto de países que, embora tenha produção científica e tecnológica sistemática, está abaixo de um possível "limiar de produção científica" que expressa a existência dessa "massa crítica". A pesquisa que este artigo encerra é uma oportunidade para investigar com mais detalhe a possível desconexão, focalizando o setor saúde.

O artigo está organizado em cinco seções além desta introdução. A segunda resume a discussão sobre o conceito de sistema de inovação em saúde, indicando como as estatísticas de artigos e patentes podem ser usadas para avaliá-lo. A terceira seção apresenta os dados internacionais, utilizando uma nova divisão de países em clusters a partir de suas produções científica e tecnológica em saúde, e discute o eventual paradoxo com os dados de patentes. A quarta seção focaliza os dados para o Brasil, identificando a desconexão em termos de distribuição geográfica. A quinta seção investiga a desconexão no nível geral da dinâmica da economia brasileira. A sexta seção conclui o trabalho.

\section{SISTEMA DE INOVAÇÃO DO SETOR SAÚDE E SUA AVALIAÇÃO QUANTITATIVA}

Em artigo anterior, Albuquerque e Cassiolato (2002) descreveram de forma detalhada as especificidades dos sistemas de inovação no setor saúde. Esta seção apenas sintetiza os principais pontos daquele artigo, contribuindo para indicar como pode ser avaliado quantitativamente esse sistema de inovação setorial. ${ }^{3}$

1 A base de dados referente ao INPI foi obtida pela Fapesp e pelo MCT; a base referente ao USPTO foi construída no CedeplarUFMG utilizando dados disponibilizados no site www.uspto.gov; os dados do ISI foram fornecidos pelo MCT (para os conjunto dos países do mundo, totais por subdisciplinas para os anos de 1981, 1991 e 2001) e foram obtidos por meio do site www.isiknowledge.org (base preparada no Cedeplar-UFMG, para os dados do Brasil entre 1990-2001). (Albuquerque, Baessa e Silva, 2005, capítulo 6).

2 Pelos dados para 1998, enquanto o limiar de produção científica está em 150 artigos por milhão de habitantes, o Brasil alcança a marca de 63 artigos por milhão de habitantes. Mesmo o Estado mais desenvolvido do País, São Paulo, está abaixo deste limiar, com 129 artigos por milhão de habitantes (dados para 1999).

3 Gadelha (2003) apresenta elaboração que enriquece a avaliação do complexo industrial do setor saúde. 


\subsection{Sistemas de inovação em países desenvolvidos}

A possibilidade de desagregação de um sistema de inovação em nível setorial é contemplada pela literatura de economia da tecnologia. ${ }^{4}$ Para o setor saúde, três pontos de partida podem ser utilizados: 1) o conceito de complexo médico-industrial (Cordeiro, 1980); 2) as evidências fortes encontradas por Hicks e Katz (1996) sobre a existência de um sistema biomédico de inovação; e 3) os estudos sobre as interações entre as universidades e as indústrias na geração das inovações médicas (Gelijns e Rosenberg, 1995).

De forma exploratória e preliminar, a Figura I apresenta o conjunto de agentes e instituições envolvidas assim como os possíveis fluxos existentes.

Figura 1 - Fluxos de informações científicas e tecnológicas no sistema de inovação do setor saúde: o caso de países com sistemas maduros

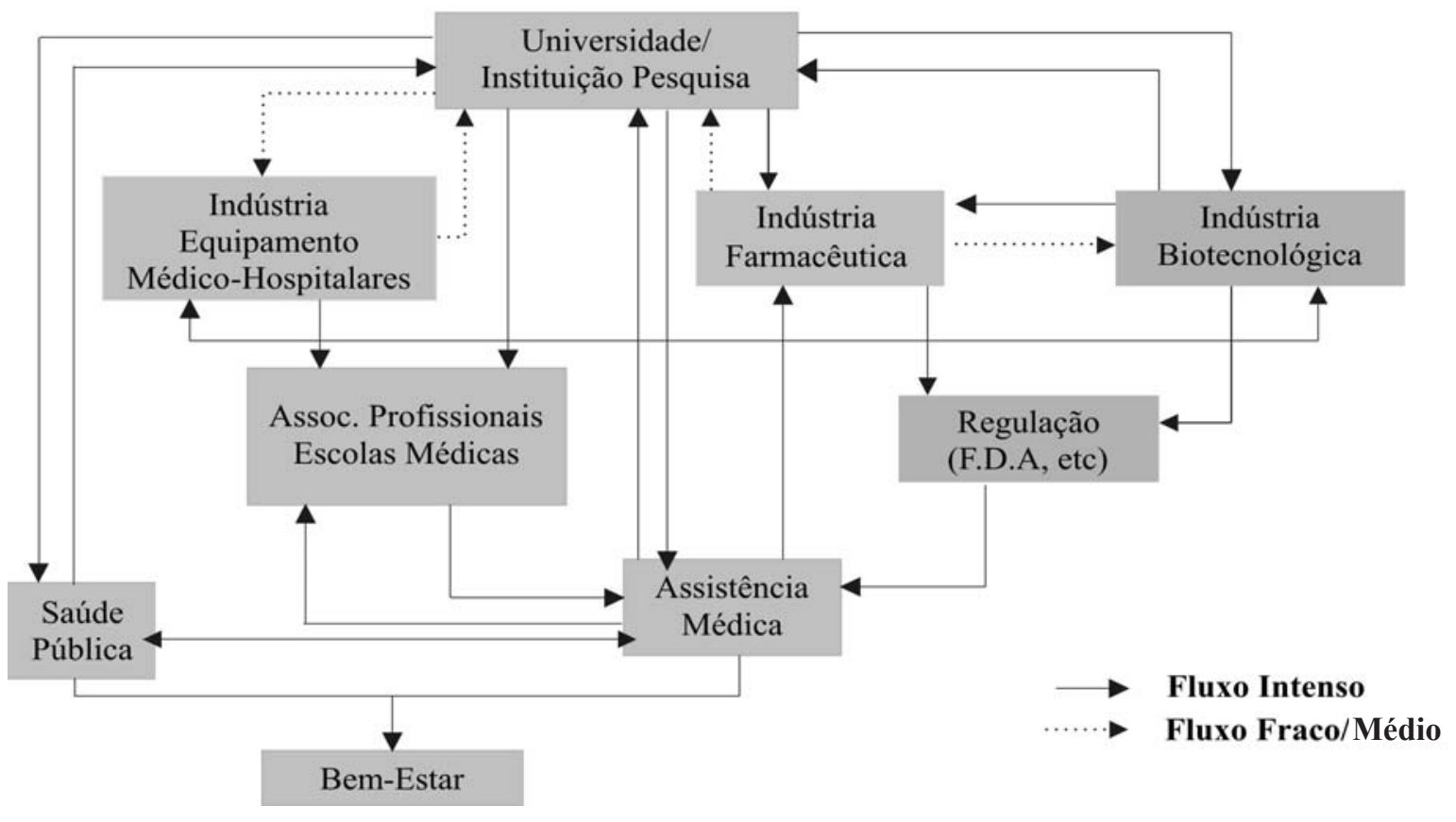

Fonte: elaboração própria, a partir de Cordeiro (1980) e Gelijns e Rosenberg (1995).

A partir da Figura 1, algumas características gerais do sistema de inovação do setor saúde podem ser apontadas:

1) O papel das universidades e instituições de pesquisa: o número de fluxos de informação científica e tecnológica que se originam ou que se destinam para essas instituições é grande. As universidades se caracterizariam como verdadeiros focos e centros de convergência de fluxos. Essa posição crucial é uma manifestação da proximidade que o progresso tecnológico do setor tem com a ciência.

2) A assistência médica envolvendo hospitais, clínicas, postos médicos etc. participa também intensamente dos fluxos, interagindo fortemente com as indústrias do setor e com a universidade.

4 Para uma discussão geral do conceito de sistemas nacionais de inovação, ver Freeman (1995) e Nelson (1993). Para discutir os sistemas setoriais, ver Breschi e Malerba (1997). 
Hospitais e centros médicos acadêmicos têm uma posição chave na implementação, difusão e melhorias incrementais de inovações. Apresentam demandas para os componentes do sistema e interagem ao longo do seu desenvolvimento.

3) Instituições de regulação (como o FDA nos Estados Unidos), associações profissionais e escolas médicas cumprem um papel de filtro das inovações geradas pelas universidades e indústrias. Essa combinação de instituições aponta o papel singular desempenhado por ambientes seletivos não-mercantis no setor saúde.

4) As indústrias têm graus diferentes de interação com as universidades. A separação entre indústrias biotecnológicas e farmacêuticas é útil em termos didáticos, mas um pouco forçada. Nos próximos anos pode haver tendência à fusão entre essas duas indústrias. ${ }^{5}$

5) A saúde pública entra com um papel importante, tendo interações diretas com as universidades e instituições de pesquisa, além de receber as inovações provenientes do complexo médico-industrial. As interações se processam de forma mediada pelo sistema de assistência médica.

6) A efetividade das inovações implementadas pela assistência médica e saúde pública repercute diretamente sobre o bem-estar da população, que é o objetivo final do conjunto do sistema de inovação da saúde. Indicadores das melhorias de bem-estar social são a ampliação da expectativa de vida (World Bank, 1993) e a redução de AVAIs (Murray e Lopez, 1996).

Essas características destacam o setor saúde como um sistema "fortemente baseado na ciência”. A literatura da Economia da Tecnologia enfatiza as diferenças intersetoriais nas relações entre ciência, tecnologia e produção. Por isso, é interessante mencionar a ênfase atribuída por acadêmicos da Economia da Tecnologia para a proximidade e para o entrelaçamento existente entre a ciência e a tecnologia no setor saúde. (Nelson, 1995; Gelijns e Rosenberg, 1995). Nelson (1995), por sua vez, confessou-se impressionado com a relevância do entrelaçamento entre ciência e tecnologia para a pesquisa e a inovação médica. Na verdade, talvez existam relações mais complexas do que as apontadas pela esquemática Figura 1: Hicks e Katz (1996) discutem o papel dos hospitais como parte do sistema de pesquisa.

\subsection{Uma pequena nota sobre estatísticas de artigos e patentes para avaliar o sistema de inovação em saúde}

Uma vez apresentada a discussão conceitual, trata-se de indicar como as estatísticas de artigos científicos e de patentes podem contribuir para uma avaliação quantitativa do sistema de inovação em saúde.

Em primeiro lugar, a produção científica (que utiliza como proxy os artigos indexados pelo ISI) refere-se fundamentalmente às universidades e institutos de pesquisa (o "topo" da Figura 1). ${ }^{6}$

5 Henderson et al. (1999), por exemplo, avaliam o impacto da revolução da biologia molecular e da biotecnologia sobre os padrões tecnológicos da indústria farmacêutica.

6 Das 105 subdisciplinas organizadas pelo ISI (conforme a base de dados cedida pelo MCT) 48 são consideradas como relacionadas à saúde por esse artigo: Anestesiologia; Biologia; Biologia celular e do Desenvolvimento; Biologia Experimental; Biologia Molecular \& Genética; Bioquímica e Biofísica; Biotecnologia e Microbiologia Aplicada; Cirurgia; Clínica em Psicologia \& Psiquiatria; Clínica Imunológica \& Doenças Infecciosas; Dentística, Cirurgia Oral e Medicina; Dermatologia; Endocrinologia, Nutrição e Metabolismo; Entomologia; Farmacologia \& Farmácia; Fisiologia; Gastroenterologia e Hepatologia; Ciência da Saúde \& Serviços; Hematologia; Imunologia; Medicina Ambiental \& Saúde Pública; Medicina Geral; Medicina Geral e Interna; Medicina Reprodutiva; Microbiologia; Neurociência \& comportamento; Neurologia; Oftalmologia; Oncologia; Oncologia \& Pesquisa sobre Câncer; Ortopedia; Otorrinolaringologia; Pediatria; Pesquisa Médica; Pesquisa Médica \& Tópicos Gerais; Pesquisa Médica, Diagnóstico \& Tratamento; Psicologia; Psiquiatria; Radiologia, Medicina Nuclear e Imagens; Reabilitação; Tecnologia Médica, Pesquisa \& Laboratórios Médicos; Reumatologia; Saúde Pública \& Ciência da Saúde; Sistema Cardiovascular \& Pesquisa em Hematologia; Sistema Cardiovascular \& Respiratório; Toxicologia; Urologia. 
Algumas empresas localizadas entre as três indústrias descritas nas "caixas" localizadas abaixo das universidades podem publicar resultados de pesquisa. Localizados na fronteira entre o sistema de inovação e o sistema de bem-estar social, os hospitais também publicam artigos.

Em segundo lugar, a produção tecnológica (que utiliza como proxy as patentes registradas no USPTO e no INPI) refere-se fundamentalmente às empresas de equipamentos médico-hospitalares, farmacêuticas e biotecnologia, conforme a Figura 1. ${ }^{7}$ Embora as firmas devam ser as principais patenteadoras, as universidades e os institutos de pesquisa também possuem patentes.

Em terceiro lugar, na interação entre ciência e tecnologia é necessário lembrar o caráter fortemente baseado na ciência do setor. (Nelson, 1995). Em termos estatísticos, pode-se supor que o setor saúde é um setor no qual a proporção artigos científicos/patentes é elevada. Para uma ilustração, basta comparar com setores caracterizados como de "baixa tecnologia", em que uma patente pode surgir sem nenhuma publicação científica para apoiá-la. Há estudos que precisam essa distinção, avaliando o peso das citações de artigos científicos em patentes: Schmoch (1997) mostra que, por esse quesito, tecnologias relacionadas à saúde (biotecnologia, medicamentos) estão entre as que mais citações têm em suas patentes.

Em quarto lugar, é importante considerar que há espaço para diversas inovações importantes para o setor que não são captadas nem por artigos nem por patentes. Referem-se a novas práticas clínicas, novas práticas de saúde pública, inovações de diagnóstico e de tratamento implementadas em hospitais. A Figura 1 é útil para ilustrar esses pontos: os fluxos entre as universidades/instituições de pesquisa e a saúde pública, a assistência médica, as escolas e associações profissionais podem muitas vezes expressar uma relação que tem produção científica na origem e inovações sem patente nas mencionadas instituições do sistema.

Essas quatro observações constituem o pano de fundo para o início da avaliação estatística. A principal referência, coerente com a discussão realizada nesta seção, é o sistema de inovação do setor saúde dos Estados Unidos. Os números são os seguintes: 53,7\% da produção científica distribuíram-se por disciplinas relacionadas à saúde, enquanto $20,7 \%$ da produção tecnológica estão em subdomínios tecnológicos relacionados à saúde. Identifica-se, assim, uma participação relativa maior da área de saúde na produção científica vis-à-vis à participação na produção tecnológica. Essa diferença pode se apoiar numa combinação entre o caráter "fortemente baseado na ciência" das tecnologias da área e o papel de alimentador direto das instituições de assistência médica e da saúde pública característico da produção científica da área.

O documento de patentes (solicitadas e concedidas) que são encontradas nos sites tanto do USPTO como do INPI contém diversas informações, possibilitando a montagem de bases de dados e sua análise. ${ }^{8}$ Entre essas informações está a classe tecnológica da patente. Existe uma classificação internacional de patentes preparada pela OMPI (www.wipo.org) que possui diversos níveis de desagregação: seções, subseções, classes e subclasses. São oito seções e mais de 600 subclasses. A classificação das patentes em subseções, por exemplo, oferece uma detalhada visão do que é a patente, a que setor se aplica etc. Para a construção das bases de dados utilizadas por este artigo, essa informação é utilizada. Entretanto, há alguns problemas. Por um lado, essa alta desagregação das subclasses dificulta a análise por separar tecnologias que são relacionadas. Por outro lado, a agregação aos níveis de seção e subseção é realizada para atender às necessidades dos escritórios de patentes e não para viabilizar análises acadêmicas do campo da economia da tecnologia.

7 Dos 30 subdomínios tecnológicos da classificação da OST (2000, p. 409), 6 são aqui considerados como “relacionados” à saúde: engenharia médica, química macromolecular, química orgânica, farmacêutico-cosméticos e biotecnologia.

8 INPI: busca por patentes de residentes. USPTO: busca por inventor, com atribuição de "nacionalidade" caso exista pelo menos um inventor do país (somente o primeiro inventor). Esse método traz problemas de dupla contagem e não segue rigorosamente a literatura. 
Para superar esses problemas, uma iniciativa do Obervatoire des Sciences et des Techniques (OST, 2000) propôs uma forma de agregação em seis domínios tecnológicos e em 30 subdomínios tecnológicos. ${ }^{9}$ A classificação proposta pela OST parte da classificação internacional da OMPI, mas os agrega com o auxílio de especialistas das diversas áreas, de forma a viabilizar informações para o formulador de políticas e para o analista da área de economia da tecnologia. O "algoritmo" da agregação, proposto pelo OST, pode ser encontrado em publicação da entidade. (OST, 2000, p. 409).

\section{O LIMIAR DE PRODUÇÃO CIENTÍFICA E CLUSTERS DE PAÍSES EM TERMOS DA PRODUÇÃO CIENTÍFICA E TECNOLÓGICA EM SAÚDE: DISCUTINDO O PARADOXO}

Para avaliar o sistema de inovação do setor saúde em nível internacional, mencionado na introdução, apresentam-se dois conjuntos de informações, ambos baseados em pesquisa de Chaves (2005): o primeiro conjunto trata da identificação de clusters de países construídos a partir de dados específicos de ciência e tecnologia em saúde; o segundo calcula os valores das médias relevantes para os países já organizados nesses clusters recém-identificados.

\subsection{Análise multivariada: clusters hierárquicos}

Segundo Everitt (1986) e Kaufman e Rousseeuw (1990), não há na literatura uma definição clara de cluster. Os conceitos são vagos e circulares, pois utilizam termos como similaridade, distância e semelhança, sem defini-los. O consenso é de que não há uma definição única para cluster. Fica a critério do pesquisador a forma de avaliar e utilizar o termo cluster. Neste artigo adota-se a definição proposta por Everitt (1986, p. 60): clusters são descritos como uma região contínua do espaço $p$ dimensional, que contém uma densidade relativamente grande de pontos, separada de outras regiões que contêm uma densidade relativamente menor de pontos.

Existem duas técnicas hierárquicas: aglomerativa e divisiva (ou de partição). Segundo Kaufman e Rousseeuw (1990):
"Elas constroem sua hierarquia em direções opostas, possivelmente gerando resul- tados bem diferentes. (...) O método aglomerativo começa quando todos os objetos estão separados (isto é, no passo zero temos n clusters). Então, em cada passo dois clusters são unidos até que somente um seja gerado. Por outro lado, o método di- visivo começa quando todos os objetos estiverem juntos (isto é, no passo zero há um cluster) e em cada passo seguinte um cluster é dividido, até que haja n clusters." (p. 44)

Neste artigo será utilizada a metodologia de clusters hierárquicos para encontrar uma tipologia entre os países em termos de sua produção científica e tecnológica, expressa por artigos e patentes, respectivamente. Assim, será possível estabelecer os países que compõem os regimes II e III relativos ao setor saúde e comparar os resultados com os do sistema nacional de inovação proposto por Silva (2003).

Em alguns casos a unidade de medida das variáveis pode alterar sensivelmente os resultados da estrutura do cluster. Por isso, os dados devem ser padronizados. (Everitt, 1986; Kaufman e Rousseeuw, 1990). Esta padronização é feita por meio da média e do desvio médio absoluto:

9 Essa classificação foi utilizada pelos Indicadores 2001. (Fapesp, 2001, Capítulo 7, seção 5). 


$$
Z_{i k}=\frac{X_{i k}-\bar{X}_{k}}{\sigma_{k}}
$$

Em que: $Z_{i k}$ possui média igual a zero e desvio médio absoluto igual a 1 ;

$\mathrm{X}_{\mathrm{k}}$ é a média e $\sigma_{\mathrm{k}}$ o desvio médio absoluto da variável $k$.

Os dados da análise de cluster são organizados em termos de $p$ variáveis e $n$ objetos. No método aglomerativo hierárquico procura-se converter os dados brutos por meio de uma medida de distância, após a padronização, gerando-se uma matriz de distâncias. A medida mais comum é a distância euclidiana, dada por:

$$
d_{i j}=\sqrt{\sum_{k=1}^{p}\left(X_{i k}-X_{j k}\right)^{2}}
$$

Em que: $\mathrm{X}_{\mathrm{ik}}$ é o valor da $\mathrm{k} t h$ variável para o ith objeto.

Finalmente, a questão da determinação do número ótimo de clusters não possui uma solução formal definitiva. Segundo Everitt (1986, p. 66), "o problema de determinar o número de clusters mais apropriado para um conjunto de dados pode ser difícil. Apesar dos numerosos debates na literatura, deve ser dito que nenhuma solução completamente satisfatória está disponivel." Neste trabalho foi estabelecido que oito é o número ideal de clusters. Com um número superior a oito, países como Taiwan, caracterizado por ter especialização em engenharia e atividades industriais, pertencente ao regime II em saúde e classificado entre os países componentes dos sistemas imaturos e sistemas maduros sem ênfase em saúde, ficaria fora do cluster 1, junto com os países do regime III, classificados como pertencentes a sistemas maduros com ênfase em saúde.

\subsection{Diferenças entre os clusters em saúde e os clusters para a produção total em ciência e tecno- logia}

Bernardes e Albuquerque (2003) identificaram três grupos de países com características semelhantes a partir das estatísticas de artigos e patentes, amplamente utilizadas na literatura como proxies de ciência e tecnologia, para o sistema nacional de inovação (NSI). A tipologia de países construída nesse artigo foi adaptada para especificar as características do sistema de inovação em saúde. Os países pertencentes ao regime I (cluster 0 - C0) não possuem produção sistemática em ciência e/ou tecnologia em saúde. Os países que compõem o regime II (cluster 1-C1) possuem sistemas de inovação imaturos e maduros sem ênfase em saúde. Finalmente, os países do regime III (cluster $>1-\mathrm{C}>1$ ) possuem sistemas maduros com ênfase em saúde.

Chaves (2005), ${ }^{10}$ utilizando dados de 2001, aplica a técnica de cluster para o setor saúde, a partir da investigação realizada por Silva (2003), que utiliza dados para 2000. Há mudanças importantes quando os dados da área de saúde são tratados isoladamente. Chaves encontra 47 países pertencentes ao regime I em saúde (Albânia e Zâmbia são exemplos), enquanto Silva (2003) encontra 41 países, quando a produção científica e tecnológica total é considerada. Bolívia, Jamaica e Azerbaijão são exemplos de países que estão no regime I de Chaves e no regime II de Silva (ver Tabela 1).

10 Chaves (2005) utiliza base de dados fornecida pelo MCT e define como do setor saúde 48 disciplinas entre 105 existentes na base utilizada (para uma lista dessas 48 disciplinas, ver Anexo, Lista I). 
Para os países do regime II, do cluster de sistemas imaturos e sistemas maduros sem ênfase em saúde, também há um crescimento no número de países quando apenas a saúde é considerada: Chaves encontra 54 países enquanto Silva encontra 52 países. O Brasil se mantém nesse cluster, ao lado dos outros três imaturos analisados em trabalho anterior (África do Sul, Índia e México). Os países do Leste Asiático passam para esse cluster (Taiwan, Coréia do Sul, Cingapura).

Finalmente, para os países do regime III, do cluster de sistemas maduros com ênfase em saúde, há uma queda na participação: 27 nos dados de Silva e 17 países para os dados de Chaves. É importante ressaltar que em 1981 e em 2001 os países pertencentes a este regime são exatamente os mesmos. ${ }^{11}$

Talvez a principal conclusão a ser tirada desses dados é relativa ao papel da especialização científica no setor saúde. Por um lado, para países acima do limiar geral de produção científica, pertencentes ao regime III, a ausência de uma boa ênfase no setor saúde pode significar um "rebaixamento" quando apenas esse setor é considerado (os melhores exemplos são os casos de Taiwan e da Coréia do Sul, indicados como não especializados em saúde). Por outro lado, mesmo uma maior especialização em saúde (Brasil comparado com Coréia do Sul) não garante a "promoção" para um cluster superior, dado o peso da ausência de superação do limiar geral de produção científica.

\subsection{Características da transição de clusters em saúde}

Ao se conjecturar sobre a existência ou não da desconexão no sistema de inovação em saúde no Brasil verifica-se que há um problema que deve ser previamente discutido: quando os dados do USPTO (nesse caso para patentes com inventores brasileiros) são analisados, a proporção relativa de patentes em saúde passa de 7\% (INPI) para 20\% (USPTO). Portanto, há um paradoxo aparente em termos da produção tecnológica do setor saúde ao compararmos os registros das duas fontes de informação. Para essa discussão, uma avaliação do cenário internacional é necessária. A identificação de limiares e de clusters de países por produção científica e tecnológica é importante.

A Tabela 1 organiza os dados para os três clusters, contribuindo para a discussão da existência ou não do paradoxo em termos dos dados de patentes no USPTO e INPI.

11 Países que pertencem ao sistema de inovação maduro com ênfase em saúde em 1981 e 2001: Finlândia, Noruega, Austrália, Canadá, Nova Zelândia, Reino Unido, Áustria, França, Bélgica, Holanda, Alemanha, Japão, Dinamarca, Suécia, Israel, Estados Unidos e Suíça. 
Tabela 1 - Total de patentes, patentes da área de saúde, total de artigos, artigos da área de saúde e participação relativa da área de saúde nas patentes e nos artigos de acordo com três "clusters" de países - 2001

\begin{tabular}{|c|c|c|c|c|c|c|}
\hline Clusters & Patente Saúde & Patente Total & Artigo Saúde & Artigo Total & \% Patente saúde & \% Artigo saúde \\
\hline CO-Média & 0 & 1,17 & 117,93 & 371,15 & 0 & 42,00 \\
\hline C0-Coef. Variação & 0 & 2,82 & 1,93 & 2,13 & 0 & 0,57 \\
\hline \multicolumn{7}{|l|}{ C0: $n$ (inicial=47) } \\
\hline C1-Média & 30,88 & 311,18 & 1710,45 & 5465,10 & 51,00 & 35,40 \\
\hline C1-Coef. Variação & 2,44 & 3,51 & 1,89 & 1,67 & 1,08 & 0,46 \\
\hline \multicolumn{7}{|l|}{ C1: $n$ (inicial=54) } \\
\hline C>1-Média & 1907,53 & 9915,94 & 24231,41 & 48046,82 & 25,00 & 50,40 \\
\hline C>1-Coef. Variação & 2,55 & 2,46 & 1,64 & 1,56 & 0,40 & 0,10 \\
\hline C>1: $n$ (inicial=17) & & & & & & \\
\hline
\end{tabular}

Fonte: Chaves (2004), utilizando dados do ISI (2003) e do USPTO (2004).

Notas: 1) Segundo Chaves (2004) os países componentes dos clusters são os seguintes: Sistemas sem Produção Sistemática em Ciência e/ou Tecnologia em Saúde (Cluster 0): Albania; Argélia; Armênia; Azerbaijão; Bolívia; Bosnia \& Herceg; Camarões; Congo, Dem Rep; Etiopia; Gana; Guiné; Haiti; Iraque; Jamaica; Quirguistão; Líbano; Lesoto; Líbia; Macedônia; Rep. Malagasi; Malawi; Mali; Mauritania; Maurício; Mongolia; Marrocos; Myanmar; Namibia; Nepal; Niger; Omã; Paquistão; Panamá; Paraguai; Portugal; Senegal; Serra Leoa; Sudão; Tanzânia; Tunisia; Emirados Árabes Unidos; Uganda; Uruguai; Usbequistão; Yemen; Zambia (os 18 países em negrito não constam na base utilizada na Tabela 1). Sistemas Imaturos e Sistemas Maduros sem Ênfase em Saúde (Cluster 1): Argentina; Belarus; Brasil; Bulgária; Chile; China; Colombia; Croácia; Cuba; Rep. Checa; Rep. Dominicana; Equador; Egito; El Salvador; Estônia; Grécia; Honduras; Hungria; Índia; Indonésia; Irã; Irlanda; Itália; Jordânia; Cazaquistão; Quênia; Kuwait; Latvia; Lituânia; Malásia; México; Nigéria; Peru; Filipinas; Polônia; Romênia; Russia; Arábia Saudita; Cingapura; Eslováquia; Eslovênia; África do Sul; Corea do Sul; Espanha; Sri Lanka; Taiwan; Tailândia; Trinidade e Tobago; Turquia; Ucrânia; Venezuela; Vietnã; Iugoslavia; Zimbabwe (os 5 países em negrito não constam na base utilizada na Tabela 1). Sistemas Maduros Com Ênfase Em Saúde (Cluster >1): Austrália; Áustria; Bélgica; Canadá; Dinamarca; Finlândia; França; Alemanha; Israel; Japão; Holanda; Nova Zelândia; Noruega; Suécia; Suíça; Reino Unico; Estados Unidos.

2) Quanto ao cluster 0 , há 10 países, dentre os 47, detentores de patentes. Nenhuma das patentes refere-se à área da saúde. Há 27 países, dentre os 47, que possuem produção científica. Todos os países possuem artigos na área da saúde com destaque para Portugal. No Haiti, $91,67 \%$ dos artigos referem-se à saúde.

Os valores encontrados para os sistemas maduros com ênfase em saúde (cluster $>1$ ) são muito próximos ao do sistema de inovação do setor saúde dos Estados Unidos, referenciado na seção 2.

Em termos absolutos, as médias das produções científica e tecnológica crescem significativamente ao se passar do cluster relativo aos sistemas imaturos e sistemas maduros sem ênfase em saúde para o cluster dos sistemas maduros com ênfase em saúde. O crescimento das médias é reflexo direto da hipótese que fundamenta a pesquisa de limiares e de clusters: uma quantidade mínima de artigos (massa crítica em termos de produção científica) é necessária para ampliar a "eficiência" na transformação do conhecimento científico em conhecimento tecnológico.

Aliás, esse fenômeno (identificado para a produção geral em ciência e tecnologia, ver Bernardes e Albuquerque, 2003) repete-se aqui: para os 17 países do cluster de sistemas maduros com ênfase em saúde são necessários, em média, 30 artigos para se gerar uma patente na área, enquanto 
que para os países do cluster de sistemas imaturos e sistemas maduros sem ênfase em saúde são necessários 120 artigos para se gerar uma patente. Ou seja, maior produção científica gera mais eficiência na produção tecnológica.

A Tabela 1 indica um comportamento interessante: países mais desenvolvidos enfatizam mais fortemente a produção científica do setor saúde. Em média, os países do cluster de sistemas maduros com ênfase em saúde dedicam $50,40 \%$ de sua produção científica ao setor saúde (valor próximo ao dos Estados Unidos - 53,7\%), média superior à do cluster de sistemas imaturos e sistemas maduros sem ênfase em saúde, que é de 35,40\%. Dada a importância do setor, a ênfase em saúde aparece desde o início, mesmo para os países componentes dos sistemas sem produção sistemática em ciência e/ou tecnologia em saúde, cuja média da produção científica em saúde é de $42 \%$.

A transição dos países pertencentes aos sistemas imaturos e sistemas maduros sem ênfase em saúde para sistemas maduros com ênfase em saúde significa um duplo movimento: por um lado cresce a produção científica de forma significativa, por outro lado também cresce a ênfase dada ao setor saúde. A persistência da Coréia do Sul e de Taiwan no cluster dos países imaturos para setor saúde (em contraste com a transição para o cluster dos países maduros para os dados como um todo) de certa forma confirma essa observação, na medida em que esses dois países realizaram apenas o primeiro movimento.

É interessante ressaltar que 16 entre os 17 países do cluster de sistemas maduros com ênfase em saúde estão entre os 21 países com índice de desenvolvimento humano superior a 0,90. (UNDP, 2001). Israel (em $22^{\circ}$ lugar) é o único da lista de países do cluster de sistemas maduros com IDH menor que 0,90 (igual a 0,893 ). Os países que não estão entre os 17 desse cluster são Luxemburgo, Islândia, Irlanda e Espanha.

A identificação dos clusters e os dados da Tabela 1 permitem responder à questão da existência ou não de um paradoxo com relação aos dados para o Brasil. A resposta é negativa, pois o Brasil se enquadra de forma razoável no padrão dos países de cluster com sistemas de inovação imaturos e maduros sem ênfase em saúde:

1) A ênfase na produção científica da área de saúde é característica do cluster de sistemas imaturos e maduros sem ênfase em saúde. O Brasil tem, segundo esses dados, 38,2\% da produção científica no setor, um pouco acima da ênfase média do setor $(35,40 \%)$, mas bem abaixo da ênfase dos países mais avançados (50,40\%);

2) A média do cluster indica que países com baixa produção tecnológica (poucas patentes no USPTO) tendem a enfatizar mais fortemente a área de saúde (11 países do cluster de sistemas imaturos e maduros sem ênfase em saúde têm participação da área de saúde igual ou maior a $50 \%)$;

3) Os valores apresentados para as participações relativas da área de saúde sugerem que à medida que os países se desenvolvem, por um lado enfatizam mais a produção científica da saúde, por outro diversificam mais a sua produção tecnológica;

4) Não tendo ultrapassado o limiar, os países do cluster de sistemas imaturos e maduros sem ênfase em saúde têm baixa eficiência na transformação de artigos em patentes: no caso brasileiro são necessários 181 artigos para gerar uma patente no setor saúde (o Brasil está acima da média do cluster 1, que é de 130 artigos por patente).

Esses quatro pontos sugerem, portanto, a inexistência de um paradoxo na comparação entre os dados internacionais (patentes no USPTO) e as patentes domésticas (INPI): o padrão do Brasil 
enquadra-se nos países de nível tecnológico similar e a identificação da desconexão entre a produção em ciência e em tecnologia pode ser investigada.

\section{OS DADOS DA PRODUÇÃo CIENTÍFICA E TECNOLÓGICA NO BRASIL: IDENTIFI- CANDO A DESCONEXÃO}

Em termos da distribuição geográfica, as patentes do período 1990-2001, referentes à base do INPI, ${ }^{12}$ estão distribuídas em 987 municípios. As patentes "relacionadas ao setor saúde" do mesmo período distribuem-se em 232 municípios. A produção científica é mais concentrada espacialmente: em 1999 foi realizada em endereços de 229 municípios. A produção científica em disciplinas relacionadas à saúde concentra-se em 170 municípios. ${ }^{13}$

Nesta seção, a desconexão é tratada tendo uma lógica espacial e geográfica, sendo que a unidade de análise é a mesorregião do IBGE. Existem 164 mesorregiões, incluindo 26 relativas a "municípios ignorados" nos estados. As regiões metropolitanas são consideradas mesorregiões.

O nível de agregação espacial em mesorregiões tem sido utilizado nas análises de especialistas em economia regional. A principal razão é a possibilidade de captar spill-overs (conforme Zitt et al., 2003) entre universidades situadas em um município (São Paulo, por exemplo) e firmas presentes em um município vizinho (Osasco, por exemplo). Para os objetivos deste artigo, o nível mesorregião é mais apropriado para a investigação da possível desconexão entre a produção científica e a tecnológica em saúde, pois a avaliação em nível do município pode deixar de captar interações existentes entre uma universidade situada em um município e firmas situadas em municípios vizinhos.

A lógica desta seção é bastante simples. Tomando a mesorregião como unidade de análise, são apresentados os dados relativos à produção tecnológica e científica. Por meio dos dados de artigos e patentes, identificam-se as regiões líderes e a concentração espacial dessas atividades. A partir dessa identificação é construída uma tabela que investiga a existência ou não de interações, comparando as disciplinas científicas líderes e os subdomínios tecnológicos líderes nas mesorregiões líderes (Tabela 2). A existência de conexão entre as produções científica e tecnológica é identificada caso haja liderança simultânea de disciplinas científicas relacionadas à saúde, por um lado, e de subdomínios tecnológicos relacionados à saúde, por outro, em uma mesma mesorregião.

A Tabela 2 realiza o "teste" da existência de conexão ou desconexão entre a produção científica e tecnológica em termos geográficos.

A Tabela 2 de certa forma reflete os dados apresentados na introdução deste artigo sobre o peso do setor saúde entre as disciplinas científicas ( $46 \%$ do total) e a debilidade entre os subdomínios tecnológicos (7\% do total).

Do lado da produção científica, em todas as mesorregiões líderes, definidas a partir dos dados de artigos e patentes (conforme Tabela 2), há pelo menos uma disciplina relacionada à saúde. Em três mesorregiões (Porto Alegre, Curitiba e Ribeirão Preto) todas as disciplinas líderes são relativas à saúde. Em outras oito mesorregiões existem duas disciplinas relacionadas à saúde. Campinas é a única região com apenas uma disciplina na área de saúde entre as líderes. A forte presença das disciplinas sobre saúde confirma, de certa forma, um viés em relação a este setor na especialização científica nacional.

12 Nesta seção é recomendável utilizar dados do INPI, pois: 1 - o número de patentes é maior; 2 - capta inovações importantes em nível nacional (mas não em nível internacional).

13 Nesta seção utiliza-se base de dados montada no Cedeplar-UFMG a partir do www.isiknowledge.org. São definidas como do setor saúde 77 disciplinas entre 169 existentes nesta base. 
Tabela 2 - Especialização científica (artigos científicos 2000) e tecnológica (patentes 1990 2001) para as doze mesorregióes líderes

\begin{tabular}{|c|c|c|c|c|c|}
\hline Mesorregião & UF & Disciplina & Artigos & Subdomínio tecnológico (OST) & Patentes \\
\hline \multirow[t]{3}{*}{ METROP. DE SAO PAULO } & SP & Bioquímica e Biologia Molecular & 172 & 29. Consumo das Famílias & 1829 \\
\hline & & Física, Multidisciplinar & 99 & 24. Manutenção-Gráfica & 1140 \\
\hline & & Neurociências & 91 & 30. Construção Civil & 800 \\
\hline \multirow[t]{3}{*}{ METROP. DO RIO DE JANEIRO } & RJ & Física, Multidisciplinar & 129 & 29. Consumo das Famílias & 355 \\
\hline & & Bioquímica e Biologia Molecular & 126 & 30. Construção Civil & 229 \\
\hline & & Parasitologia & 88 & 24. Manutenção-Gráfica & 176 \\
\hline \multirow[t]{3}{*}{ METROP. DE BELO HORIZONTE } & MG & Ciência Veterinária & 69 & 29. Consumo das Famílias & 167 \\
\hline & & Parasitologia & 41 & 30. Construção Civil & 138 \\
\hline & & Química, Multidisciplinar & 33 & 07. Análise-Mensuração-Controle & 127 \\
\hline \multirow[t]{3}{*}{ METROP. DE PORTO ALEGRE } & RS & Neurociências & 63 & 29. Consumo das Famílias & 242 \\
\hline & & Bioquímica e Biologia Molecular & 42 & 16. Trabalho com Materiais & 118 \\
\hline & & Genética e Hereditariedade & 29 & 24. Manutenção-Gráfica & 92 \\
\hline \multirow[t]{3}{*}{ METROP. DE CURITIBA } & PR & Neurociências & 28 & 29. Consumo das Famílias & 274 \\
\hline & & Psquiatria & 26 & 24. Manutenção-Gráfica & 122 \\
\hline & & Biotecnologia e Microbiologia Aplicada & 16 & 30. Construção Civil & 101 \\
\hline \multirow[t]{3}{*}{ CAMPINAS } & SP & Engenharia Química & 79 & 29. Consumo das Famílias & 136 \\
\hline & & Bioquímica e Biologia Molecular & 74 & 24. Manutenção-Gráfica & 94 \\
\hline & & Química, Física & 72 & 30. Construção Civil & 79 \\
\hline \multirow[t]{3}{*}{ DIST. FEDERAL } & DF & Bioquímica e Biologia Molecular & 27 & 29. Consumo das Famílias & 60 \\
\hline & & Botânica & 19 & 07. Análise-Mensuração-Controle & 30 \\
\hline & & Física, Multidisciplinar & 18 & 26. Transportes & 28 \\
\hline \multirow[t]{3}{*}{ RIBEIRAO PRETO } & $\mathrm{SP}$ & Bioquímica e Biologia Molecular & 32 & 29. Consumo das Famílias & 84 \\
\hline & & Farmacologia e Farmácia & 29 & 25. Aparelhos Agrícolas e & 52 \\
\hline & & Neurociências & 29 & 24. Manutenção-Gráfica & 34 \\
\hline \multirow[t]{3}{*}{ NORTE CENTRAL PARANAENSE } & PR & Bioquímica e Biologia Molecular & 18 & 29. Consumo das Famílias & 76 \\
\hline & & Botânica & 13 & 24. Manutenção-Gráfica & 36 \\
\hline & & Agricultura Multidisciplinar & 12 & 26. Transportes & 33 \\
\hline \multirow[t]{3}{*}{ PIRACICABA } & $\mathrm{SP}$ & Química Analítica & 24 & 25. Aparelhos Agrícolas e & 66 \\
\hline & & Dentística, Cirurgia Oral e Medicina & 19 & 29. Consumo das Famílias & 42 \\
\hline & & Bioquímica e Biologia Molecular & 17 & 14. Procedimentos Técnicos & 24 \\
\hline \multirow[t]{3}{*}{ METROP. DE RECIFE } & $\mathrm{PE}$ & Física da Matéria Condensada & 15 & 29. Consumo das Famílias & 30 \\
\hline & & Bioquímica e Biologia Molecular & 12 & 24. Manutenção-Gráfica & 24 \\
\hline & & Botânica & 11 & 30. Construção Civil & 20 \\
\hline \multirow[t]{3}{*}{ GRANDE FLORIANOPOLIS } & SC & Engenharia Elétrica e Eletrônica & 26 & 29. Consumo das Famílias & 27 \\
\hline & & Farmacologia e Farmácia & 21 & 24. Manutenção-Gráfica & 21 \\
\hline & & Bioquímica e Biologia Molecular & 17 & 07. Análise-Mensuração-Controle & 19 \\
\hline
\end{tabular}

Fonte: Elaboração própria a partir de INPI (2002, 2003), ISI (2003), RAIS (1997).

Em termos da produção tecnológica, a liderança do subdomínio “consumo das famílias” é geral (primeiro lugar em onze mesorregiões), expressando o padrão de especialização tecnológica do País. ${ }^{14}$ É necessário destacar que nenhuma mesorregião tem um subdomínio tecnológico relacionado à saúde entre os subdomínios líderes.

Comparando os dois lados, encontra-se uma desconexão: a saúde está entre as disciplinas principais das mesorregiões líderes, mas está ausente entre os subdomínios tecnológicos dessas

14 Conforme OST (2000, p. 325), o consumo doméstico é o padrão de especialização da América latina. Em sintonia com este resultado, Albuquerque, Baessa e Silva (2005, cap. 6, seção 3.2.2, p. 6-17/6-19) demonstram que o consumo doméstico é o padrão de especialização do Brasil. 
mesorregiões. O resultado apresentado a partir da análise de distribuição espacial confirma a conjectura inicial elaborada com os dados gerais: há evidências fortes de desconexão entre as produções científica e tecnológica no setor saúde.

\section{A DEBILIDAdE do SISTEMA DE INOVAÇÃo EM SAÚdE, O PAPEL DE PATENTES DE NÃO-RESIDENTES E PROBLEMAS NA BALANÇA COMERCIAL}

Com a hipótese da desconexão não tendo sido refutada até este ponto da análise, esta seção tem o objetivo de investigar como a desconexão é resolvida na dinâmica mais geral da economia brasileira. A forma de solução pode representar um problema adicional para o sistema de inovação em saúde, repercutindo sobre a intensidade dos fluxos tecnológicos característicos do setor.

Há algo característico de um país com relativo atraso tecnológico e econômico. Este aspecto está relacionado às particularidades da inserção internacional do País nos fluxos tecnológicos.

Dois aspectos podem ser visualizados com estatísticas disponíveis: patentes de não-residentes (INPI) e comércio exterior (MDIC, por meio da base Alice-SECEX).

\subsection{A ênfase em saúde nas patentes de não-residentes}

Em relação às patentes de não-residentes depositadas no INPI, elas podem sinalizar políticas de empresas transnacionais, com ou sem subsidiárias instaladas no Brasil, em relação ao mercado nacional.

As patentes de não-residentes são muito importantes nas estatísticas brasileiras: elas representam cerca de 60,2\% do total das patentes depositadas no INPI entre 1990 e 2001. Em uma investigação sumária dessas patentes de não-residentes, foram encontradas 26.333 patentes nos subdomínios relacionados à saúde, entre as 80.204 patentes de não-residentes constantes dessa base de dados (esta base é a utilizada em trabalho anterior, ver Biazzi et al., 2001). Ou seja, 32,84\% das patentes de não-residentes são relacionadas à saúde. Uma proporção quase cinco vezes superior à encontrada entre as patentes de residentes para um período similar. Esse dado sugere um papel importante para empresas transnacionais nas atividades relacionadas ao setor saúde.

\subsection{Debilidade no sistema de inovação, déficit na balança comercial}

Em relação às atividades de comércio exterior, estudos têm demonstrado a posição problemática do Brasil em áreas de alta tecnologia. E o setor saúde é um setor de alta tecnologia. Dados coletados por Sarti e Sabbatini (2003, p. 416 e 420) apresentam o déficit do País em mercadorias descritas como "produtos farmacêuticos e médicos" (déficit comercial de US $\$ 1,59$ bilhões em 2001) e como "instrumentos médicos" (déficit comercial de US\$ 0,14 bilhões em 2001). Para Sarti e Sabbatini, os dois grupos de mercadorias são classificados como de "alta intensidade" tecnológica.

Esses dados sugerem que as deficiências existentes na estrutura industrial do sistema de inovação do setor saúde (de certa forma captadas pelas estatísticas de patentes: a parcela de 7\%, que representa a saúde na produção tecnológica do País) são supridas por importação. Por um lado, as mercadorias classificadas como "aparelhos para medicina, cirurgia, odontologia e veterinária" (mercadorias com o código NCM iniciadas por 9018, no capítulo 90) apresentaram déficit na balança comercial brasileira de US $\$ 1,11$ bilhão entre 1999 e 2003. É importante destacar que o 
nível de sofisticação para esses produtos é elevado (aparelhos de ressonância magnética, ecógrafos Doppler, ultra-sons, câmaras gama, endoscópios, aparelhos para cirurgia que operem por laser, rins artificiais etc). Por outro lado, para produtos menos sofisticados (agulhas, seringas) e aparelhos para odontologia (instrumentos, aparelhos, brocas etc.) o Brasil apresenta saldo positivo de aproximadamente US\$92,5 milhões.

Para mercadorias classificadas como produtos farmacêuticos (capítulo 30 da classificação NCM) existe um déficit na balança comercial brasileira de US\$ 8,85 bilhões entre 1996 e 2003. É importante comentar a posição deficitária do País em termos de vacinas: dos 21 diferentes tipos de mercadorias iniciadas com o nome "vacina", o Brasil é superavitário em apenas dois tipos (vacina veterinária contra a febre aftosa e vacinas anticatarral e antipiogênico, em doses).

Uma observação sumária desses dados sugere uma hipótese a ser testada em outro trabalho: as áreas onde inexistem patentes são atendidas por importação, donde parece existir uma correlação entre a inexistência de capacitação industrial-tecnológica e déficit na balança comercial. Caso esta correlação seja comprovada, uma implicação em termos de política pública é apresentada: o fortalecimento da dimensão industrial do sistema de inovação do setor saúde contribui para a melhoria da balança comercial brasileira.

Em síntese, a desconexão existente entre a produção científica e tecnológica no País é preenchida por importação de mercadorias, indicando que produtos são desenvolvidos sem envolvimento de pesquisadores brasileiros (na matriz ou em outros centros de desenvolvimento no exterior) e que podem ou não ser fabricados no País. A forte presença do setor saúde entre as patentes de não-residentes é uma questão a ser posteriormente explorada: empresas transnacionais desenvolvem produtos em laboratórios no exterior, patenteiam no INPI e não acrescentam capacidade inovadora além da capacidade de produzir determinados produtos. Isto significa que as subsidiárias de transnacionais localizadas no País poderiam contribuir mais para o amadurecimento do sistema de inovação do setor saúde do que têm feito até aqui - outro tema para políticas públicas.

\section{COMENTÁRIOS FINAIS}

Partindo do conceito de sistema de inovação do setor saúde e apresentando uma forma de avaliação quantitativa desse sistema por estatísticas de artigos e patentes (de forma a captar as diversas dimensões do sistema, graficamente ilustradas na Figura 1) este artigo sumaria os principais resultados encontrados.

Na pesquisa, identificou-se a posição internacional do País em termos de ciência e tecnologia em saúde, colocando-o em uma situação intermediária no cluster de países pertencentes a sistemas imaturos e maduros sem ênfase em saúde. Essa posição é fortemente influenciada pela situação geral da ciência e da tecnologia no País, em particular pela posição do Brasil abaixo de um "limiar de produção científica" identificado em outros estudos.

$\mathrm{Na}$ avaliação dos dados internacionais, percebe-se a existência de um duplo movimento à medida que os países passam para níveis mais desenvolvidos: por um lado, cresce a produção científica de forma significativa, por outro, também cresce a ênfase dada ao setor saúde. A consciência desse duplo movimento ressalta a possibilidade, aberta ao Brasil, de construir um processo de catching up que enfatize o setor saúde.

A hipótese da desconexão entre a produção científica e tecnológica não é descartada ao longo deste texto, na medida em que a avaliação da distribuição espacial das produções científica e tecnológica também não refuta essa hipótese. Considera-se que é necessário reconhecer um ponto 
de partida importante para a construção do sistema de inovação do setor saúde no País, e que não é possível desconhecer a profundidade do desafio perante os formuladores de políticas públicas: as conexões potenciais devem ser construídas ao mesmo tempo que a própria produção científica nacional deve crescer, pois esse crescimento parece ser uma condição para conexões estáveis entre as duas dimensões.

Porém, a conclusão da avaliação preliminar aqui apresentada não pode ser pessimista, por duas razões. Por um lado, a existência de desconexão indica uma linha para intervenção das políticas públicas, no sentido do aproveitamento mais efetivo das contribuições potenciais do setor científico do País. A busca da construção de conexões talvez seja a principal orientação para as políticas públicas, que devem gerar formas criativas para incentivar o setor produtivo ligado à saúde. Um subproduto importante de políticas para a construção do sistema setorial de inovação em saúde é uma melhora, no médio prazo, da balança comercial do País. Por outro lado, a abrangência das especializações regionais indica a capacidade do País para enfrentar o "mosaico epidemiológico" característico do Brasil. O perfil epidemiológico do Brasil se distingue dos países avançados pela presença dos "problemas persistentes", incluindo doenças tropicais, e também dos países mais pobres pela presença das "epidemias emergentes", envolvendo doenças não-transmissíveis. ${ }^{15}$ Por isso, o Brasil é um país que necessita de um sistema de saúde que dê conta, simultaneamente, de lidar com doenças parasitárias e com doenças degenerativas. Portanto, o perfil epidemiológico do País apresenta demandas muito especiais sobre o sistema de saúde e sobre a infra-estrutura científica.

Quanto a uma agenda de pesquisa, é importante insistir no caráter "preliminar" dessa avaliação. Uma avaliação mais completa necessita de articulação entre os dados de ciência e tecnologia com informações sobre o desempenho produtivo de setores industriais relacionados à saúde, dados a respeito da prestação de serviços médicos, estatísticas de saúde e de bem-estar social. Estudos de caso voltados para casos particulares de interação entre a dimensão científica e tecnológica são necessários também, pois é possível a existência de conexões parciais e localizadas que não sejam captáveis pelos dados aqui apresentados (e pela forma como foram tratados).

Pesquisas nessa linha são indispensáveis para escrutinar, com detalhe e abrangência, a hipótese central deste trabalho sobre a desconexão existente entre a produção científica e tecnológica no sistema de inovação do setor saúde no País.

\section{REFERÊNCIAS BIBLIOGRÁFICAS}

Albuquerque, E. M.; Baessa, Adriano; Silva, Leandro Alves. Atividade de patenteamento no Brasil e no exterior. In: FAPESP. (org.), Indicadores de ciência, tecnologia e inovação em São Paulo - 2004. São Paulo, 2005, v. 1, p. 1-37.

Albuquerque, E. Patentes e atividades inovativas: uma avaliação preliminar do caso brasileiro. In: Viotti, E.; Macedo, M. Indicadores de ciência, tecnologia e inovação no Brasil. Campinas: Editora Unicamp, 2003.

Albuquerque, E.; Cassiolato, J. E. As especificidades do sistema de inovação do setor saúde. Revista de Economia Política, v. 22, n. 4, p. 134-151, 2002.

Bernardes, A.; Albuquerque, E. Cross-over, thresholds and interactions between science and technology: lessons for less-developed countries. Research Policy, v. 32, n. 5, p. 865-885, 2003.

15 Buck et al. (1988) distinguem três estágios na evolução dos padrões de doenças: 1) estágio marcado por doenças infecciosas associadas à pobreza, má nutrição, falta de saneamento etc; 2) doenças degenerativas como doenças cardíacas e câncer; 3) problemas derivados de poluição ambiental e problemas em famílias, comunidades e locais de trabalho que levam à violência, abuso de drogas, alcoolismo etc. Os países desenvolvidos teriam passado por esses três estágios ao longo de um século, enquanto os países em desenvolvimento devem enfrentá-las de uma só vez. Daí a menção ao "verdadeiro mosaico epidemiológico". 
Biazzi, E.; Albuquerque, E. Transnational corporations and patenting activities in Brazil: data description and statistical tests about the relative internalization of technological activities. Economia Aplicada, v. 5, n. 2, p. 407-431, abr-jun. 2001.

Breschi, S.; Malerba, F. Sectoral innovation systems. In: Edquist, C. (ed.), Systems of innovation: technologies, institutions and organizations. London: Pinter, 1997.

Buck, C.; Llopis, A.; Nájera, E.; Terris, M. The challenge o epidemiology: issues and selected readings. Washington: PAHO/WHO, 1988.

Chaves, C. V. As interações entre o setor saúde e a saúde mental a partir das estatísticas de ciência e tecnologia. 2005. 154 f. Tese (Doutorado em Economia), Centro de Desenvolvimento e Planejamento Regional - CEDEPLAR-UFMG. Belo Horizonte.

Cordeiro, H. A indústria da saúde no Brasil. Rio de Janeiro: Graal, 1980.

Everitt, B. Cluster analysis. New York: Halstet, 1986. 136p.

Freeman, C. The "National System of Innovation" in historical perspective. Cambridge Journal of Economics, v. 19, n. 1, 1995.

Fundação de Amparo à Pesquisa do Estado de São Paulo - FAPESP. Indicadores de ciência, tecnologia e inovação em São Paulo. São Paulo: FAPESP, 2001.

Gadelha, C. O complexo industrial da saúde e a necessidade de um enfoque dinâmico na economia da saúde. Ciência e Saúde Coletiva, v. 8, n. 2, p. 521-535, 2003.

Gelijns, A.; Rosenberg, N. The changing nature of medical technology development. In: Rosenberg, N.; Gelijns, A.; Dawkins, H., Sources of medical technology: universities and industry (Medical innovation at the crossroads, v. 5). Washington: National Academy, 1995.

Henderson, R.; Orsenigo, L.; Pisano, G. The pharmaceutical industry and the revolution in molecular biology: interactions among scientific, institutional, and organizational change. In: Mowery, D.; Nelson, R., Sources of industrial leadership: studies of seven industries. Cambridge: Cambridge University, 1999.

Hicks, D.; Katz, J. Hospitals: the hidden research system. Science and Public Policy, v. 23, n. 5, p. 297-304, Oct. 1996.

Kaufman, L., Rousseeuw, P. J. Finding groups in data: an introduction to cluster analysis. New York: John Wiley, 1990. 342p.

Murray, C. J. L., Lopez, A. D. Global and regional descriptive epidemiology of disability: incidence, prevalence, health expectancies and year lived with disability. In: Murray, C. J. L., Lopez; A. D. (eds.), The global burden of disease: a comprehensive assessment of mortality and disability from disease, injuries, and risk factors in 1990 and projected to 2020. Cambridge, MA: Harvard University, 1996, cap.4, p. 201-247.

Nelson, R. The intertwining of public and proprietary in medical technology. In: Rosenberg, N.; Gelijns, A.; Dawkins, H., Sources of medical technology: universities and industry (Medical innovation at the crossroads, v. 5). Washington: National Academy, 1995.

Observatoire des Sciences et des Techniques. Science \& Technologie: indicateurs 2000. Paris: Economica, 2000.

Sarti, F.; Sabbatini, R. Conteúdo tecnológico do comércio exterior brasileiro. In: Viotti, E.; Macedo, M. Indicadores de ciência, tecnologia e inovação no Brasil. Campinas: Editora Unicamp, 2003.

Science Citation Index. Institute for Science Information. Web of Science 2002, 2003. Disponível em: http://www.webofscience.com/.

Schmoch, U. Indicators and the relations between science and technology. Scientometrics, v.38, n.1, p.103116, 1997.

Silva, L. Padrões de interação entre ciência e tecnologia. 2003. Dissertação (Mestrado), Centro de Desenvolvimento e Planejamento Regional - CEDEPLAR-UFMG. Belo Horizonte.

World Bank. World development report 1993: investing in health. Oxford: Oxford University, 1993. 
UNDP. Making new technologies work for human development. Human Development Report 2001. New York: Oxford University. Disponível em www.undp.org.

United States Patentes and Trademark Office - USPTO. 2002, 2003. Disponível em: http://www.uspto. gov.

Zitt, M.; Ramanana-Rahary, S.; Bassecoulard, E.; Laville, F. Potential science-technology spillovers in regions: an insight on geographic co-location of knowledge activities in the EU. Scientometrics, v. 57, n. 2, p. 295-320, 2003. 